\title{
Topological Characteristics of Planar Linkage Including Platform Type Robots
}

\author{
Ashok Dargar \\ Department of Mechanical Engineering, School of Engineering, Sir Padampat Singhania University,NH-76, Udaipur- 313601, India \\ *Corresponding author: dargarashok@rediffmail.com
}

Copyright (C) 2014 Horizon Research Publishing All rights reserved.

\begin{abstract}
In order to select the best possible chain or mechanism at the conceptual stage of design, the designer should be able to read the characteristics of the kinematic chains based on their topology. To accomplish this it is only necessary to associate logically certain characteristics of a chain to perform a task, with the structure and then generalize. Based on this belief in the present work chains are modeled as a system of springs connected in series to reveal some of the characteristics like stiffness and compactness. The concepts developed are applied to platform type robots, which are gaining in importance.
\end{abstract}

Keywords Kinematic Chain (KC), Link Value, Degree Of Freedom, Stiffness, Compactness

\section{Introduction}

There are two types of kinematic chains, i.e. serial and parallel. In serial choice is limited while in parallel, there are distinct structures such as 16 in eight links single degree of freedom, 40 in nine links 2 degree of freedom, 98 in 10 links 3 degree of freedom and more than 6000 single DOF with twelve links $[1,2]$. Through knowledge of kinematic chains is essential for a designer and persistent efforts are made to know about kinematic chains as much as possible. The complexity of kinematic chains increases with the number of the links. For the chains with the same number and type of links, the structure or the topology of the chain is expected to play a prominent role. Despite availability of many chains with distinct structure there are no guidelines to select the best possible chain for the specified task such as function generation, path generation or robot manipulator application, etc. Until now, most of the work is directed to study isomorphism among chains and to know the type of freedom [3-7] work relating to this aspect is hardly seen in the literature. Some work reported earlier [8-10] deals with the dimensional aspects and not with the structural influence. Rao and Rao $[11,12]$ proposed link-loop Hamming values for rating of the kinematic chains. Rao [13] compare the kinematic chains for characteristics like mechanical advantages, compactness and stiffness. Rao and Jagdeesh [14] compare the chains from the viewpoint of balancing and their expected dynamic behavior. Rao $[15,16]$ presented a pseudo genetic algorithm for evaluation of kinematic chains. Rao [17] using the concept of fuzzy logic proposed a numerical measure to compare the chain characteristics like symmetry, parallelism and mobility. Srinath and Rao [18] proposed the concept of correlation to evaluate the performance of the chains.

Exact static and dynamic behavior of linkages in an absolute sense cannot be predicted unless the link shapes, dimensions, masses and their distribution etc., are known. However, it should be possible to compare different chains with same number of links and joints for their expected behavior on the basis of their structure, without having to perform the actual static and dynamic analysis. Structure of a chain depends upon the link assortment, i.e., type, number and their adjacency. The author believes that the structure of a chain reveals its capabilities and limitations accordingly an attempt was made to compare the chains from the viewpoint of stiffness and compactness extending the concepts proposed by Rao [13] these concepts to compare platform-type robots, which are gaining importance, is dealt with.

\section{Definitions of Terminology}

The following definitions are to be understood clearly before applying this method. Various definitions with their abbreviations are given below.

(i) Degree of link: A numerical value for the link, based on its connectivity to other links therefore quaternary link has degree equal to four and ternary link has degree equal to three.

(ii) Joint value: It is the ratio of summation of degree of all the links connected at the joint to the number of links connected at the joint (type of joint). it is denoted by $J_{v}$ and computed by Eq.(1).

$$
\mathrm{J}_{\mathrm{v}}=\frac{\sum \text { Degree of all the connected links }}{\text { number of links connected at the joint }}
$$




\section{Basic Theory}

\subsection{Stiffness}

The actual stiffness of a link or a member of a chain depends upon its dimensions, elasticity and connectivity or supports. Since a chain is modeled as a system of springs connected in series, if all the links are stiffer the chain can be expected to posses' greater stiffness and this will lead to lighter chains or links. When the chains are to be compared on the basis of their structure, the modulus of elasticity and dimensions of all the links can be considered to be same so the stiffness of the link is the function of its supports only. A member can be considered to have been elastically supported by as many other members as there are joints. Also, a member becomes stiffer if the numbers of supports are more. Likewise the stiffness of a link increases with the connectivity of the link itself and of the adjacent links. Based on this theory the stiffness of a link is defined as the sum of all the joint values of that link. As a chain is modeled like a system of springs connected in series, the stiffness of a chain is computed by Eq. (2)

$$
\frac{1}{S}=\frac{1}{S 1}+\frac{1}{S 2}+\frac{1}{S 3}+\ldots .+\frac{1}{S n}
$$

Here $S_{n}$ is the stiffness of the $n^{\text {th }}$ link of the chain. To illustrate the above two distinct six link chains are considered.

For Stephenson's chain Fig. 1(a)

$$
\frac{1}{\mathrm{~S}}=\frac{1}{7.5}+\frac{1}{5}+\frac{1}{7.5}+\frac{1}{5}+\frac{1}{4.5}+\frac{1}{4.5}
$$

For Watt's chain Fig. 1(b)

$$
\frac{1}{S}=\frac{1}{8}+\frac{1}{5}+\frac{1}{7.5}+\frac{1}{5}+\frac{1}{4.5}+\frac{1}{4.5}
$$

Thus the stiffness value of the Stephenson's chain Fig. 1(a) and of Watt's chain Fig. 1(b) are 0.9/1.11 and 0.878 /1.1027 respectively, indicating the Stephenson chain is stiffer or more rigid compared to the Watt chain. Also, consider two eight link chains of Fig. 2(a) and 2(b), stiffness value of them are 0.7438 and 0.7686 respectively indicating that chain of Fig. 2(a) is structurally more stiffer or rigid.

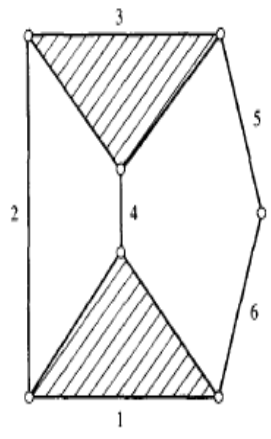

(a)

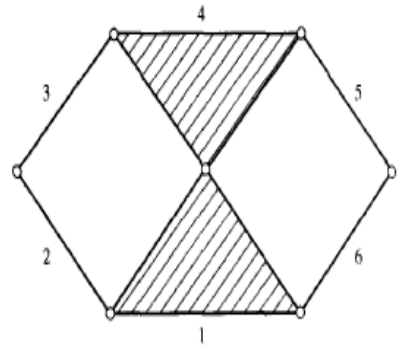

(b)
Figure 1. (a) Stephenson chain; (b) Watt chain

\subsection{Compactness}

The knowledge of structural compactness of a chain at least in comparative sense is always desirable. A chain in which links and joints are close to one another may be considered as a compact chain. Closeness of any two links and joints may be indicated by the distance between them respectively. In graph theory, the distance between two vertices is defined as the least number of edges that fall between the two vertices out all possible paths between them. Adapting this concept, the distance between the two links is equal to the least number of joints that separate them and distance between the two joints is equal to the least number of links that separate them. Two distance matrices link distance matrix $D_{1}$ and joint distance matrix $D_{2}$ are written for every chain. For link distance matrix $\mathrm{D}_{1}, \mathrm{D}_{\mathrm{ij}}=$ least number of joints between links $\mathrm{i}$ and $\mathrm{j}$ and $\mathrm{D}_{\mathrm{ii}}=0$ and for joint distance matrix $\mathrm{D}_{2}, \mathrm{D}_{\mathrm{ij}}=$ least number of links between joints $\mathrm{i}$ and $\mathrm{j}$ and $\mathrm{D}_{\mathrm{ii}}=0$.

For example, distance matrices for the two distinct six link chains is given as

For Stephenson's chain Fig. 1(a)

$$
\mathrm{D}_{1}=\left[\begin{array}{llllll}
0 & 1 & 2 & 1 & 2 & 1 \\
1 & 0 & 1 & 2 & 2 & 2 \\
2 & 1 & 0 & 1 & 1 & 2 \\
1 & 2 & 1 & 0 & 2 & 2 \\
2 & 2 & 1 & 2 & 0 & 1 \\
1 & 2 & 2 & 2 & 1 & 0
\end{array}\right]
$$

$$
\mathrm{D} 2=\left[\begin{array}{lllllll}
0 & 1 & 2 & 2 & 1 & 2 & 2 \\
1 & 0 & 1 & 2 & 2 & 1 & 2 \\
2 & 1 & 0 & 1 & 2 & 1 & 2 \\
2 & 2 & 1 & 0 & 1 & 2 & 2 \\
1 & 2 & 2 & 1 & 0 & 2 & 1 \\
2 & 1 & 1 & 2 & 2 & 0 & 1 \\
2 & 2 & 2 & 2 & 1 & 1 & 0
\end{array}\right]
$$

The sum of all the elements in the $\mathrm{D}_{1}$ matrix is called as the link distance value of the chain, which for this chain is 46 and the sum of all the elements in the $\mathrm{D}_{2}$ matrix is called as the joint distance value of the chain, which for this chain is 66.

For Watt's chain Fig. 1(b)

$$
\begin{gathered}
\mathrm{D}_{1}=\left[\begin{array}{llllll}
0 & 1 & 2 & 1 & 2 & 1 \\
1 & 0 & 1 & 2 & 3 & 2 \\
2 & 1 & 0 & 1 & 2 & 3 \\
1 & 2 & 1 & 0 & 1 & 2 \\
2 & 3 & 2 & 1 & 0 & 1 \\
1 & 2 & 3 & 2 & 1 & 0
\end{array}\right] \\
\mathrm{D}_{2}=\left[\begin{array}{lllllll}
0 & 1 & 2 & 2 & 2 & 1 & 1 \\
1 & 0 & 1 & 2 & 3 & 2 & 2 \\
2 & 1 & 0 & 1 & 2 & 2 & 1 \\
2 & 2 & 1 & 0 & 1 & 2 & 1 \\
2 & 3 & 2 & 1 & 0 & 1 & 2 \\
1 & 2 & 2 & 2 & 1 & 0 & 1 \\
1 & 2 & 1 & 1 & 2 & 1 & 0
\end{array}\right]
\end{gathered}
$$

The sum of all the elements in the $\mathrm{D}_{1}$ matrix is called as the link distance value of the chain, which for this chain is 50 
and the sum of all the elements in the $\mathrm{D}_{2}$ matrix is called as the joint distance value of the chain, which for this chain is 66.

In order to assess the compactness of a chain, a numerical measure is necessary and to compute the numerical measure a compactness index $\mathrm{C}$ is proposed, defined as the sum of link distance value and joint distance value. For example the compactness C of Stephenson's chain Fig. 1(a) is $46+56=$ 102 and for Watt chain Fig. 1(b) is 50+ 66 = 106 indicating that Stephenson's chain is structurally more compact. Also, consider two eight link chains of Fig. 2(a) and 2(b), value of the compactness index $C$ are respectively $96+157=253$ and $100+162=262$ respectively indicating that chain of Fig. 2(a) is structurally more compact.

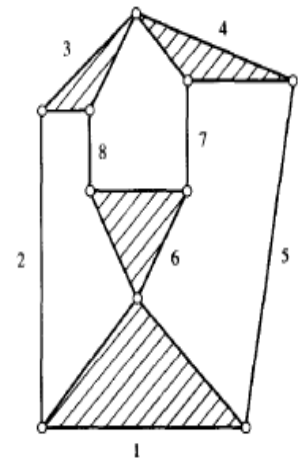

(a)

Figure 2. .(a) and (b) Two eight link 1 d. o. f. Chains

\section{Application to platform- type robots}

In the recent past open- chain linkages have received greater attention for robot arms. Each joint in these robot arms is actuated independently. While possessing many advantages, such as large work space and maneuverability, they do suffer from disadvantages like less rigidity, accumulation of mechanical errors from shoulder to the endeffectors, control problems, etc. The alternative to the open chain robot arms is the in- parallel actuator arrangement often referred to as the platform-type robot as it has greater rigidity, lightness, load carrying capacity and the actuators can be attached to a fixed base [12-15]. The disadvantages of the platform -type robot is that the forward kinematic problem is more complex than the inverse kinematics of open chain robots but some closed form solutions are presented for forward kinematic analysis of platform type robot. In what follows only planar robots are dealt with.

To illustrate the above discussed concepts, let us consider two 3 d.o.f. eight link chains of Fig. 3 (a) and 3(b).

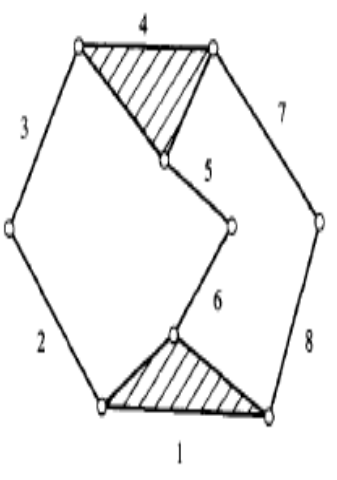

(a)

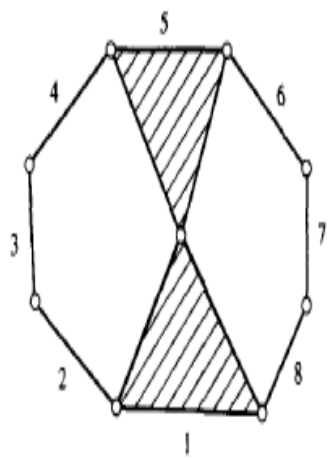

(b)
Figure 3. (a) and (b) Two eight link 3 d. o. f. chains

For the chain of Fig. 3(a)

$$
\begin{gathered}
\frac{1}{s}=\frac{1}{7.5}+\frac{1}{4.5}+\frac{1}{4.5}+\frac{1}{7.5}+\frac{1}{4.5}+\frac{1}{4.5}+\frac{1}{4.5}+\frac{1}{4.5} \\
\mathrm{D} 1=\left[\begin{array}{llllllll}
0 & 1 & 2 & 3 & 2 & 1 & 2 & 1 \\
1 & 0 & 1 & 2 & 3 & 2 & 3 & 2 \\
2 & 1 & 0 & 1 & 2 & 3 & 2 & 3 \\
3 & 2 & 1 & 0 & 1 & 2 & 1 & 2 \\
2 & 3 & 2 & 1 & 0 & 1 & 2 & 3 \\
1 & 2 & 3 & 2 & 1 & 0 & 3 & 2 \\
2 & 3 & 2 & 1 & 2 & 3 & 0 & 1 \\
1 & 2 & 3 & 2 & 3 & 2 & 1 & 0
\end{array}\right]
\end{gathered}
$$

The link distance value of the chain is 108 .

$$
\mathrm{D}_{2}=\left[\begin{array}{lllllllll}
0 & 1 & 2 & 3 & 2 & 1 & 1 & 2 & 3 \\
1 & 0 & 1 & 2 & 3 & 2 & 2 & 3 & 2 \\
2 & 1 & 0 & 1 & 2 & 3 & 3 & 2 & 1 \\
3 & 2 & 1 & 0 & 1 & 2 & 3 & 2 & 1 \\
2 & 3 & 2 & 1 & 0 & 1 & 2 & 3 & 2 \\
1 & 2 & 3 & 2 & 1 & 0 & 1 & 2 & 3 \\
1 & 2 & 3 & 3 & 2 & 1 & 0 & 1 & 2 \\
2 & 3 & 2 & 2 & 3 & 2 & 1 & 0 & 1 \\
3 & 2 & 1 & 1 & 2 & 3 & 2 & 1 & 0
\end{array}\right]
$$

The joint distance value of the chain is 138 .

So the value of stiffness and compactness of this chain are 0.625 and $108+138=246$ respectively.

For the chain of Fig. 3(b)

$$
\begin{aligned}
\frac{1}{S} & =\frac{1}{8}+\frac{1}{4.5}+\frac{1}{4}+\frac{1}{4.5}+\frac{1}{8}+\frac{1}{4.5}+\frac{1}{4}+\frac{1}{4.5} \\
\mathrm{D}_{1} & =\left[\begin{array}{lllllllll}
0 & 1 & 2 & 2 & 1 & 2 & 2 & 1 \\
1 & 0 & 1 & 2 & 2 & 3 & 3 & 2 \\
2 & 1 & 0 & 1 & 2 & 3 & 4 & 3 \\
2 & 2 & 1 & 0 & 1 & 2 & 3 & 3 \\
1 & 2 & 2 & 1 & 0 & 1 & 2 & 2 \\
2 & 3 & 3 & 2 & 1 & 0 & 1 & 2 \\
2 & 3 & 4 & 3 & 2 & 1 & 0 & 1 \\
1 & 2 & 3 & 3 & 2 & 2 & 1 & 0
\end{array}\right]
\end{aligned}
$$




$$
\mathrm{D}_{2}=\left[\begin{array}{lllllllll}
0 & 1 & 2 & 3 & 2 & 1 & 1 & 2 & 3 \\
1 & 0 & 1 & 2 & 3 & 2 & 2 & 3 & 2 \\
2 & 1 & 0 & 1 & 2 & 3 & 3 & 2 & 1 \\
3 & 2 & 1 & 0 & 1 & 2 & 3 & 2 & 1 \\
2 & 3 & 2 & 1 & 0 & 1 & 2 & 3 & 2 \\
1 & 2 & 3 & 2 & 1 & 0 & 1 & 2 & 3 \\
1 & 2 & 3 & 3 & 2 & 1 & 0 & 1 & 2 \\
2 & 3 & 2 & 2 & 3 & 2 & 1 & 0 & 1 \\
3 & 2 & 1 & 1 & 2 & 3 & 2 & 1 & 0
\end{array}\right]
$$

The joint distance value of the chain is 140 .

So the value of stiffness and compactness of this chain are 0.610 and $110+140=250$ respectively. Thus, the chain of Fig. 3(a) is more rigid and compact as compared to chain of Fig. 3(b) and should be selected for platform type robot application.

\section{Conclusions}

Based on the analysis of the above discussed chains and many more chains not included in the paper, the following conclusions are drawn.

(i). The lesser the distance values of a chain or inversion, greater is the compactness.

(ii). Compact chains are stiffer also and hence to become too light to carry the same loads. This comparison is permissible among chains consisting of an equal number and type of links.

(iii). Loss of motion and power increases with the number of joints, hence compact chains or inversions are better from this viewpoint.

(iv). Compact chain has less joint interaction, are more accurately controlled.

\section{REFERENCES}

[1] K. Hain, Applied kinematics, 2nd edn. (New York: McGraw Hill Companies, inc. 1967).

[2] C. H. Suh, C.W. Radcliffe, Kinematics and Mechanism Design (New York: John Wiley and Sons, Inc.1964).

[3] Dargar, A., Khan, R. A., and Hasan, A., Some New Codes for Isomorphism Identification among Kinematic Chains and their Inversions, International Journal of Mechanisms and Robotic Systems, 1 (1), 49-67, (2013).
[4] Dargar, A., Khan, R. A., and Hasan, A., A Method for Identification of Isomorphism and Structural Properties of Kinematic Chains, International Journal of Materials and Structural Integrity , 5 (4), 376-388, (2011).

[5] Dargar, A., Khan, R. A., and Hasan, A. Application of Link Adjacency Values to Detect Isomorphism among Kinematic Chains, International Journal of Mechanics and Materials in Design, 6, 157-162, (2010) .

[6] Dargar, A., Khan, R. A., and Hasan, A., Mobility Analysis of Kinematic Chains, Kathmandu University Journal of Science, Engineering and Technology, 6(1) 25-32, 2010.

[7] Dargar, A., Khan, R. A., and Hasan, A., Identification of Isomorphism among Kinematic Chains and Inversions using Link Adjacency Values, International Journal of Mechanical and Materials Engineering , 4 (3), 309-315, 2009.

[8] F. Freudenstein, 'Approximate synthesis of four- bar linkages' ASME J. Mech. Des. 77, 853-855, (1995):.

[9] F. Freudenstein, 'Structural error analysis in plane kinematic synthesis' J. Eng. Ind., 81 (1), 15-22, (1959).

[10] P. S. Rose, G. N. Sandor, 'Direct analytical synthesis of four bar function generators with optimal structure error' J. Eng. Ind. 95 (2), 563-571, (1973).

[11] A. C. Rao, C. N. Rao, 'Loop based pseudo hamming values $-\mathrm{I}$ : Testing isomorphism and rating of kinematic chains' Mech. Mach. Theory, 28 (1), 113-127, (1993).

[12] A. C. Rao, C. N. Rao, 'Loop based pseudo hamming values -II; Inversions preferred frames and actuators' Mech. Mach. Theory 28 (1), 129-143, (1993).

[13] A. C. Rao, 'Topological characteristics of linkage mechanisms with particular reference to platform' Mech. Mach. Theory, 30 (1), 30-39, (1995).

[14] A. C. Rao, A. Jagadeesh, 'Structure based dynamic characteristics of planar linkages including platform type robots' Journal of Robotic Systems, 14 (8), 621-629, (1997).

[15] A. C. Rao, 'A genetic algorithm for topological characteristics of kinematic Chains' ASME J. Mech. Des. 122, 228-231, (2000):.

[16] A. C. Rao, 'Pseudogenetic algorithm for evaluation of kinematic chains' Mech. Mach. Theory 41, 473-485, (2005).

[17] A. C. Rao, 'Application of fuzzy logic for the study of isomorphism, inversions, symmetry, parallelism and mobility in kinematic chains' Mech. Mach. Theory 35, 1103-1116, (2000).

[18] A. Srinath, A. C. Rao, 'Correlation to detect isomorphism, parallelism and type of Freedom' Mech. Mach. Theory 41, 646-655, (2006): 